\title{
Non-linear cosmological power spectra in real and redshift space
}

\author{
A. N. Taylor ${ }^{1 \star}$ and A. J. S. Hamilton ${ }^{2 \star}$ \\ IInstitute for Astronomy, Unisersity of Edinburgh, Royal Observaton;, Blackford Hill, Edinburgh EH9 $3 H J$ \\ 2 JILA and Department of Astrophysical, Planetary and Atmospheric Sciences, Box 4\$0, University of Colorado, Boulder, CO 80309, USA
}

Accepted 1996 April 29. Received 1996 January 30

\begin{abstract}
A B STRACT
We present an expression for the non-linear evolution of the cosmological power spectrum based on Lagrangian trajectories. This is simplified using the Zel'dovich approximation to trace particle displacements, assuming Gaussian initial conditions. The model is found to exhibit the transfer of power from large to small scales expected in self-gravitating fields. Some exact solutions are found for power-law initial spectra.

We have extended this analysis into redshift space and found a solution for the non-linear, anisotropic redshift-space power spectrum in the limit of plane-parallel redshift distortions. The quadrupole-to-monopole ratio is calculated for the case of power-law initial spectra. We find that the shape of this ratio depends on the shape of the initial spectrum, but when scaled to linear theory depends only weakly on the redshift-space distortion parameter, $\beta$. The point of zero-crossing of the quadrupole, $k_{0}$, is found to obey a simple scaling relation and we calculate this scale in the Zel'dovich approximation.

This model is found to be in good agreement with a series of $N$-body simulations on scales down to the zero-crossing of the quadrupole, although the wavenumber at zero-crossing is underestimated. These results are applied to the quadrupole-to-monopole ratio found in the merged QDOT plus 1.2-Jy. IRAS redshift survey. Using a likelihood technique we have estimated that the distortion parameter is constrained to be $\beta>0.5$ at the 95 per cent level. Our results are fairly insensitive to the local primordial spectral slope, but the likelihood analysis suggests $n=-2$ in the translinear regime. The zero-crossing scale of the quadrupole is $k_{0}=0.5 \pm 0.1 \mathrm{~h} \mathrm{Mpc}^{-1}$ and from this we infer that the amplitude of clustering is $\sigma_{8}=0.7 \pm 0.05$.

We suggest that the success of this model is due to non-linear redshift-space effects arising from infall on to caustics and is not dominated by virialized cluster cores. The latter should start to dominate on scales below the zero-crossing of the quadrupole, where our model breaks down.
\end{abstract}

Key words: cosmology: theory - large-scale structure of Universe.

\section{INTRODUCTION}

The Newtonian analysis of linear growth of perturbations in an expanding universe is a well-understood problem (Peebles 1980; Efstathiou 1990). The extension of this into the non-linear regime has proven more difficult, owing to the strong mode coupling that arises in gravitational collapse, and most progress has been made through the use of $N$-body simulations.

Actial observations of galaxies in redshift space are further complicated (and made more interesting) by redshift distortions, caused by peculiar velocities of galaxies along the line of sight. Again. the linear problem is relatively well understood. Kaiser (1987) has shown that linear redshift distortions take their simplest form when

•E-mail: ant@roc.sc.uk (ANT); ajsh@dark.colorado.edu (AJSH) expressed in Fourier space, at least if structure is far from the observer so that the distortions are essentially plane-parallel. Here the redshiftspace Fourier modes are related to the real-space ones by

$\delta^{3}(k)=\left(1+\beta_{\mu_{k}^{2}}^{2}\right) \delta(k)$

where a superscript s denotes a redshift-space quantity, $\mu_{k}$ is the cosine of the angle between the wavevector $k$ and the line of sight, and $\beta$, the redshift distortion parameter, is the dimensionless growth rate of growing modes in linear theory, which is related to the cosmological density $\cap$ by (Peebles 1980)

$\beta=\frac{\Omega^{0.6}}{b}$

in the standard pressureless Friedmann cosmology with massto-light bias $b$. It is through measuring the distortion parameter 
B that one hopes to measure the cosmological density parameter, $\Omega$.

Kaiset's formula (1) is valid only in the lineai limit, and for plane-parallel distortions, neither of which approximations is well satisfied in reality. Several authors have now addressed the problem of generalizing Kaiser's formula to the case of radial distortions, while retaining the assumption of linearity (Fisher, Scharf \& Lahav 1994; Heavens \& Taylor 1995; Fisher el al. 1995; Zaroubi \& Hoffman 1996; Ballinger, Heavens \& Taylor 1995; Hamilton \& Culhane 1996).

The main purpose of the present paper is to extend the analysis of redshift distortions into the non-linear regime, setaining the planeparaliel approximation for simplicity. Our approach to the problem is motivaled by the consideration that the density in redshift space may appear highly non-linear even when the density in real space is only mildly non-linear. For example. a region that in real space is just tuming around, a mildly non-linear condition, appears in redshift space as a caustic, a surface of infinite density, which is thoroughly non-linear. This leads us first to work in Lagrangian space (Section 2.1), and secondly to adopt the Zel dovich (1970) approximation (Sections 2.2 and 3 ). The Zel'dovich approximation is in effect linear theory expressed in Lagrangian space, inasmuch as it approximutes the trajectories of particles as straight lines with (comoving) displacements growing according to linear theory.

Our approach follows that of Tajlor (1993), who studied the nonlinear evolution of the power spectrum. Comparable approaches have been used by Bond \& Couchman (1987) to evolve the galaxy angular correlation function, by Mann. Heavens \& Peacock (1993) to evolve the real-space correlation function of clusters, and by Schneider \& Bartelmann (1995) to evolve the real-space (unredshifted) power spectrum. Our approach differs from that of Hivon et al. (1995), who applied a perturbation expansion in Lagrangian space to second order to calculate the redshift-space skewness.

For simplicity, we assume throughout this paper that the density field is unbiased, $b=1$. Generally, the effect of evolution, if continuity is assumed (which is a fundamental assumption of this paper), is to tend to drive the bias factor towards unity. Continuity implies that the ratio of galaxy 10 matter density $(1+\delta) /\left(1+\delta_{M}\right)$ remains constant in Lagrangian elements, and if (somehow) a linear bias $\delta=b \delta_{M}$ is established at some early time when $\delta$ and $\delta_{M}$ are both small, then the ratio of galaxy to matter density must be close to unity, $\left(1+b \delta_{M}\right) /\left(1+\delta_{M}\right)-1$. Il follows that the bias will be close to unity at later, non-linear epochs when $\delta_{M}$ is no longer small. Conversely, if bias is in fact important at the present, non-linear epoch (as may well be the case), then it must be that the assumption of continuity must break down in the not too distant past. Indeed, it may be that continuity is violated on an ongoing basis. In the present paper we choose to ignore this thorny problem, and simply assume an unbiased density field.

We begin in Section 2 by deriving equations which relate the Lagrangian and Eulerian descriptions of density, and we calculate a general expression for the evolution of the power spectrum. We invoke the Zel'dovich approximation and Gaussian initial condi. tions and consider some of the general features of the resulting nonlinear power spectrum. Some exact solutions for initially powerlaw spectra are derived. In Section 3 we calculate the power spectrum in redshift space, again in the Zel'dovich approximation, and obtain a number of analytic and numerical results for the observationally interesting ratio of quadrupole-to-monopole power. In Section 4, we compare the predictions of the Zel'dovich approximation with $\boldsymbol{N}$-body simulations, and we apply our findings to analyse the quadrupole distortion measured in the QDOT plus 1.2-Jy redshift survey. We summarize our conclusions in Section 5.

\section{REAL-SPACE POWER SPECTRA}

\subsection{Clustering in the Lograngian frame}

In the Lagrangian theory of fluid mechanics, the central variable is the displacement vector field, tather than the density field of Eulerian space. This displacement field, $\xi(q . t)$, maps particles from their initial Lagrangian coordinate, $q$, to the Eulerian-space coordinate, $x$. via the telation ${ }^{1}$

$x(q, t)=q+\xi(q . t)$

where $\xi(q . t)$ is the integral of the velocity field along the world-line of the particle:

$\xi(q, t)=\int^{\prime} d t^{\prime} v\left[x\left(q, t^{\prime}\right), t^{\prime}\right]$

The advantage of the Lagrangian formalism for gravitational collapse was first pointed out in the seminal work by Zel dovich (1970). Because the coordinate system of Lagrangian space is nonlinear, moving with the particles themselves. this approach takes the analysis of perturbation grout th of the density field into the nonlinear regime while conserving mass densily.

However, the most useful observable quantity in cosmology is the Eulerian density field, $\rho(x)$, inferred either from variations in the galaxy distribution, or indirectly from distortions in the Hubble flow, from gravitational lensing, or from the microwave background. Continuity, along with the assumption of uniform initial density, implies that the relation between the overdensity $\delta(x)=[\rho(x)-\bar{\rho}] / \bar{\rho}$ in Eulerian space and the Lagrangian displacement field $\xi(q)$ is

$\delta(x)=\int \mathrm{d}^{3} q \delta_{D}[x-q-\xi(q)]-1$.

where $\delta_{D}(x)$ is the Dirac delta function. The Fourier transform

$\delta(k)=\int \mathrm{d}^{3} x \mathrm{e}^{\mathrm{ik} \cdot \mathrm{x}} \delta(x)$

of the overdensity $\delta(x)$, equation (5), is

$\delta(k)=\int d^{3} q e^{i k \cdot q}\left(e^{i k \cdot t}-1\right)$.

which relates the Fourier space Eulerian density field $\delta(k)$ to the Lagrangian displacement field $\boldsymbol{\xi}(\boldsymbol{q})$.

The correlation function of Fourier modes defines the power spectrum $P(k)$ :

$\left\langle\delta(k) \delta^{*}\left(k^{\prime}\right)\right\rangle=(2 \pi)^{3} P(k) \delta_{\mathrm{D}}\left(k-k^{\prime}\right)$,

where the angular brackets denote ensemble averaging and the Dirac delta function arises from translational invariance. Trans. forming to differential and centre-of-mass coordinates, we find that as a result of translational invariance only the differential terms survive. We can then express the, power spectrum by the integral equation

$P(k)=\int d^{3} q e^{i k \cdot q}\left(\left\langle e^{i k \cdot d k}\right\rangle-1\right)$,

where the term $\left(e^{i k \cdot s \xi}\right\}$ is identified as the generating function of the differential displacement vector field, $\Delta \xi(q)$, of points separated by

'Throughout this paper we take the coordinates $x$ and $q$ to be comoving coordinates, defined relative to the genetal Hubble expansion of the Universe. flui

the

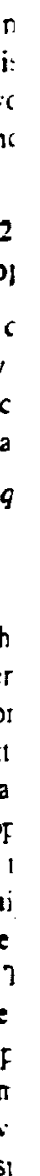

hi
or
n
if
he
he
Li

hi

$\downarrow_{L j}$

. 
distance $q=q_{1}-q_{2}$ :

$\Delta \xi(q)=\xi\left(q_{1}\right)-\xi\left(q_{2}\right)$.

Equation (9) for the power spectrum is valid at all times, not merely in the linear regime. However, in order to solve this completely one needs to have an expression for the displacement generating function and its evolution. In practice we shall have to approximate this. In the next section we use equation (9) to calculate the evolution of the power spectrum in the Zel'dovich approximation, under the assumption of random Gaussian initial conditions.

\subsection{Evolution of the power spectrum in the Zel'dovich approximation}

A careful analysis of the Zel dovich approximation has been given by Hui $\&$ Bertschinger (1995), who consider it in the context of local theories of gravity. For our purposes we only need the result that the particle displacement field scales according to linear theory:

$$
\begin{aligned}
\boldsymbol{\xi}(q . t) & =D(t) \xi_{\mathrm{L}}(q) \\
& =\frac{i D(t)}{(2 \pi)^{3}} \int d^{3} k \frac{k}{k^{2}} \delta_{L}(k) e^{-i k \cdot q},
\end{aligned}
$$

where $\xi_{L}(q)$ is the linear displacement field, and $\delta_{L}(k)$ the linear density field. defined at some suitably early time. This approximation is then a first-order theory in the displacement vector field, extrapolated to arbitrary later times. By construction the Lagran. gian coordinate system preserves mass, but in the Zel'dovich approximation at the expense of not satisfying the Euler equation. In the Lagrangian frame the density field is usually evolved along fluid streamlines, $\delta[q(x), t]$, but equation (5) allows one to calculate the density in Eulerian coordinates.

The assumption of a Gaussian initial fluctuation field implies that the initial displacement field, $\xi_{L}$, was Gaussian. The Zel'dovich approximation (11) further implies that the displacement field $\xi(q)$ remains Gaussian for all time. Equation (9) for the power spectrum involves the Lagrangian generating function of the differential displacement field, which for a Gaussian vector field is

$$
\begin{aligned}
\left\langle e^{i k \cdot \Delta k^{2}}\right\rangle & =\exp \left(-k_{i} k_{j}\left(\Delta \xi_{i} \Delta \xi_{j}\right) / 2\right) \\
& =\exp \left(-k_{i} k_{j}\left[\psi_{i j}(0)-\psi_{i j}(q)\right]\right) .
\end{aligned}
$$

where $\psi_{i j}(q)=\left\langle\xi_{i}\left(q_{1}\right) \xi_{j}\left(q_{2}\right)\right\rangle$ is the displacement correlation function. In the Zel'dovich approximation, the displacement correlation function $\downarrow_{i j}$ grows as the square of the growth factor $D(t)$ in the nonlinear as well as linear regimes:

$$
\psi_{i j}(q, t)=[D(t)]^{2} \Downarrow_{L i j}(q) \text {. }
$$

The linear correlation function $\psi_{L j}(g)$ of displacements is related to the linear power spectrum $P_{L}(k)$ by

$$
\begin{aligned}
\psi_{L j}(q) & =\left(\xi_{L i}\left(q_{1}\right) \xi_{L i}\left(q_{2}\right)\right) \\
& =\frac{1}{(2 \pi)^{3}} \int d^{3} k \frac{k_{i} k_{j}}{k^{4}} P_{L}(k) e^{-i k \cdot q} .
\end{aligned}
$$

which at zero separation, $q=0$, is

$\psi_{L j}(0)=\left\langle\xi^{2}\right\rangle \delta_{i j}=\frac{\delta_{i j}}{3(2 \pi)^{3}} \int d^{3} k k^{-2} P_{L}(k)$

where $\left(\xi^{2}\right)$ is the one-dimensional dispersion of displacements.

Inserting the Gaussian generating function (12) into equation (9) for the power spectrum yields (Taylor 1993)

$P(k)=\int d^{3} q e^{i k \cdot \cdot}\left\{e^{-k_{1} k_{j}\left(\psi_{y}(0)-\psi_{y}(q)\right]}-1\right\}$
This equation (16), along with equations (13) and (14), provides a non-linear mapping between the initial and evolved power spectra, where the degree of evolution is controlled by the linear amplitude, $L(t)$, of perturbations.

The unit term on the right of the integrand of equation (16), which arises from subtraction of the mean density, yields a delta function at $k=0$ on integration. The unit term can be ignored for $k \neq 0$, and we tacitly drop it hereafter. Nevertheless, it can help in numerical integrations to retain the unit term when $k$ is small (linear regime).

So far we have not assumed that the density field is isotropic, although we have assumed that it is homogeneous. In Section 3 we. will apply the power spectrum equation (16) in redshift space, where the density field is not isotropic. In the remainder of the present section we assume that the density field is unredshifted and isotropic.

For an isotropic density field, the displacement correlation function $\psi_{i j}(\boldsymbol{g})$ resolves into irreducible components parallel and perpendicular to the pair separation $q$ :

$\psi_{i j}(q)=\psi_{i}(q) \hat{q}_{i} \dot{q}_{j}+\downarrow_{\perp}(q)\left(\delta_{i j}-\hat{q}_{i} \hat{q}_{j}\right)$.

where

$\psi_{i}(q)=\frac{[D(t)]^{2}}{2 \pi^{2}} \int \mathrm{d} k P_{\mathrm{L}}(k)\left[j_{0}(k q)-2 \frac{j_{1}(k q)}{k q}\right]$

and

$\psi_{-}(g)=\frac{[D(t)]^{2}}{2 \pi^{2}} \int d k P_{L}(k)\left[\frac{j_{1}(k q)}{k q}\right]$.

For a general potential flow, as here, the parallel variance $\psi$ is related to the perpendicular variance $\psi_{\perp}$ by (Monin \& Yaglom 1975; Gorski 1988)

$\psi(q)=\frac{\mathrm{d} q \psi_{i}(q)}{\mathrm{d} q}$

which can also be derived from equation (14) with the definitions (17). It is convenient to define the differential displacement covariances $\psi_{+}$and $\psi_{-}$by

$\psi_{+}(q)=\psi_{-}(0)-\psi_{-}(q)$,

$\psi_{-}(q)=\psi_{-}(q)-\psi_{k}(q)$.

which are related by

$\psi_{-}(q)=\frac{q d \psi_{+}(q)}{d q}$

The (isotropic) power spectrum (16) can then be written in terms of $\psi_{+}$and $\psi_{-}$:

$P(k)=\int d^{3} q e^{i k q-k^{2}\left(\psi_{+}+\psi_{-} \mu^{2}\right)}$,

where $\mu=\hat{k} \cdot \dot{q}$. Integrating equation (24) over the azimuthal angle of $q$ about the wavevector $k$ is trivial, and further integration over $\mu$ yields

$P(k)=\operatorname{Re} \int_{0}^{x} 2 \pi q^{2} \mathrm{~d} q e^{-k^{2}\left(\psi_{+}+\psi_{-}\right)} F\left(k q, k^{2} \psi_{-}\right)$.

where $F(A, B)$ is the function

$F(A, B)=\frac{\pi^{1 / 2}}{B^{1 / 2}} \exp \left(B-\frac{A^{2}}{4 B}\right) \operatorname{erf}\left(B^{1 / 2}-\frac{i A}{2 B^{1 / 2}}\right)$

with erf (z) the error function (Abramowitz \& Stegun 1968)

$\operatorname{erf}(z)=\frac{2}{\pi^{1 / 2}} \int_{0}^{z} d r e^{-r^{2}}$.

The error function erf $(z)$ for complex $z$ is available in programs 


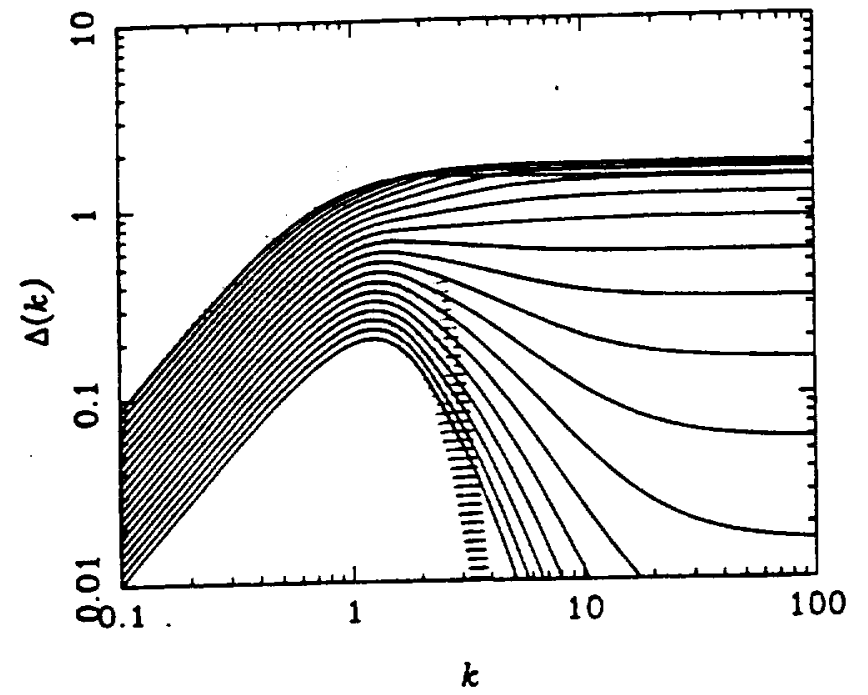

Figure 1. The evolution of the dimensionless amplitude $\Delta_{L}(k)=k^{3:} \exp \left(-k^{2} / 2\right)$ in the Zel dovich approximation for random Gaussian initial conditions. The solid lines represent the non-linear pow'er spectrum and the dotted lines represent the linear initial spectrum.

such as MAthemiatica (Wolfram Research). Equation (25) reduces the evaluation of the power spectrum to a single integral over separation 9 . Excepting some simple cases (Section 2.4), this integral must be done numerically.

The integrand in equation (25) oscillates rapidly when $\mathrm{kq}$ is large and real, which can make the integral difficult to evaluate (Schneider \& Bartelmann 1995). One way to resolve the difficulty is to continue the $\psi_{x}(q)$ analytically into the complex plane, and to shift the path of integration over $q$ into the upper complex plane, which converts the oscillations into exponential decay. Some experimentation may be required to determine a good integration path, along which the integrand is well-behaved. For example, in the case of an initially Gaussian power spectrum, Section 2.3 below; we found it satisfactory to integrate first along the real axis $10 \mathrm{~g}-\min (2 D, 1 / k)$, and then to complete the integration along a straight line slanted upwards in the complex plane, at angle $\pi / 6$ from the real line. Variants on this path, with or without the initial segment along the real axis, and with orher slant angles, worked in other cases.

\subsection{Example: a Gaussian power spectrum}

As an example, in Fig. 1 we show the Zel'dovich evolution of a power spectrum that is initially Gaussian,

$P_{L}(k)=2 \pi^{2} \exp \left(-k^{2}\right)$.

The quantity plotied is the dimensionless amplitude $\Delta(k)$, the square root of the dimensionless power spectrum defined by (Peacock 1992)

$\Delta^{2}(k)=\frac{k^{3} P(k)}{2 \pi^{2}}$.

Fig. 1 shows clearly the two main features of non-linear growth, the amplification of small-scale power from the formation of caustics, and the restriction on the growth of intermediate-scale power imposed by the positive density constraint in voids.

Fig. 1 also illustrates the limitations of the Zel'dovich approximation at later stages. While in reality the small-scale density contrast increases following the collapse of structures, in the Zel'dovich approximation structures expand back outwards after collapse. Thus in Fig. 1 the amplitude $\Delta(k)$ reaches a maximum of order unity as a function of time, and thereafter declines. In reality, the amplitude $\Delta(k)$ would be expected to increase monotonically with time.

\subsection{Solutions for initial power-law spectra}

For initially power-law spectra, $P_{L}(k) \propto k^{n}$, convergent results are attained in the Zel'dovich approximation for power spectrum indices in the range $-3<n<-1$. For $n \leq-3$, the differential acceleration berween particle pairs, hence the differential displace. ment field, receives divergent contributions from large scales, while, for $n \geq-1$, the acceleration diverges on small scales. In the latter case, non-linear processes are expected to intervene so as to truncate the divergence, but we do not consider such truncations here.

For initially power-law spectra the differential displacement covariances are power laws: $\psi_{2} \propto q^{-n-1}$. It is convenient here to normalize the growth factor, $D(t)$, so that

$\psi_{+}(q)=D^{2} q^{-n-1}$

(a normalization based, for example, on the power spectrum is less convenient because it leads to divergence in the displacement covariance for $n=-3$ and -1 ). The relation (23) implies

$\psi_{-}(q)=(-n-1) \psi_{+}(q)$.

With the normalization (30), the initial linear power spectrum $P_{\mathrm{L}}(k)$ is [note that $P_{L}(k)$ is defined without the factor $D^{2}$, so $P(k)=D^{2} P_{L}(k)$ in the linear regime]

$P_{\mathrm{L}}(k)=4 \pi(2-n) \sin [(-n-1) \pi / 2] \Gamma(1-n) k^{n}$

which is positive over the interval $-3<n<-1$, going through zero at $n=-3$ and -1 .

In the Zel'dovich approximation, initially power-law power spectra evolve in a self-similar fashion:

$\Delta^{2}(k, D)=\Delta^{2}\left(D^{2} k^{n+3}\right)$.

The self-similar evolution of scale-free initial conditions in the case of a flat, $\Omega=1$, universe is familiar from the BBGKY hierarchy (Peebles 1980). In the Zel'dovich approximation, the property of self-similarity extends also to non-critical universes.

We now give exact solutions for the cases $n=-1,-2$, and -3 in the Zel'dovich approximation.

\section{$2.4 .1 n=-1$}

In the limiting case $n \rightarrow-1$, the displacement field has an incoberent Gaussian nature. This arises because the incipient divergence of acceleration on small scales causes particles at finite separations to acquire essentially random velocities relative 10 eacb other. However, if particles in an initially uniform field move about randomly, the resulting field continues to remain uniform. Therefore the power spectrum should remain infinitesimal even when the displacement covariance grows to a finite value. Mathematically, the linear power spectrum (32) goes to zero in the limit $n \rightarrow-1$.

In teality, non-linear evolution will in this case invalidate the linear extrapolation of displacements assumed by the Zel'dovich approximation. Nevertheless, it is interesting to consider $n=-125$ a limiting case. Defining the small positive quantity $\nu$ by

$v=-n-1$,

which tends to zero as $n \rightarrow-1$, one finds that for $\psi_{+}=D^{2} q^{\prime}$, 
equation (30), the dimensionless power spectrum, $\Delta^{2}$, evolves as $\Delta^{2}(k, D)=3 \nu D^{2} k^{2} e^{-D^{2} k^{2}}$.

The power spectrum (35) is that of an $n=-1$ Gaussian field, modulated by a random Gaussian distribution of displacements with a one-dimensional one-point dispersion of $D$.

Athough the power spectrum is itself infinitesimal, the ratio $P_{2}^{s} / P_{0}^{\prime}$ of quadrupole-to-monopole redshift power, which we shall consider in more detail later, is well-defined and finite in the limit $n \rightarrow-1$.

\section{$2.4 .2 n=-2$}

For an initial power-law spectrum of index $n=-2$, the integral (24) can be done analytically. Here $\psi_{+}=\psi_{-}=D^{2} q$, and the dimensionless power spectrum evolves as

$\Delta^{2}(k, D)=\frac{16 D^{2} k}{\pi\left(1+4 D^{4} k^{2}\right)^{2}}\left[1+\frac{3 \pi D^{2} k}{4\left(1+4 D^{4} k^{2}\right)^{1 / 2}}\right]$.

In the linear regime $\Delta^{2}=16 D^{2} k / \pi$, while in the highly non-linear regime $\Delta^{2} \propto\left(D^{2} k\right)^{-3}$.

\section{$2.4 .3 n=-3$}

In the limiting case $n \rightarrow-3$, differential accelerations between pairs are dominated by tidal contributions generated on large scales, inducing motions which appear locally like anisotropic Hubble flows. The displacement covariances are $\psi_{+}=D^{2} q^{2}, \psi_{-}=2 \psi_{+}$. The integral (24) can again be done analytically, yielding

$\Delta^{2}(k, D)=\frac{e^{\left.-1 / 12 D^{2}\right)}}{(12 \pi)^{1 / 2} D^{3}}$.

Here the power spectrum remains a power law, $P(k) \propto k^{-3}$, at all times. As argued by Schneider \& Bartelmann (1995), the persistence of the $k^{-3}$ form results from the dominance of caustics in the density distribution. The amplitude initially increases, from an exponentially tiny value, as more regions reach the point of collapse, but then reaches a maximum, at $D^{2}=1 / 18$, and subsequently declines as $D^{-3}$ as regions pass through the point of collapse and continue streaming outward.

We note that equation (37) also gives the asymprotic form of the evolution of the power spectrum in the Zel'dovich approximation on small scales whenever the spectral index is less than -3 on small scales. This is true for example in the case of the Gaussian power spectrum $P_{L}(k)=2 \pi^{2} e^{-k^{2}}$ considered in Section 2.3. In this case, ms differential displacements berween pairs are proportional to their separation on small scales. Define

$\sigma^{2}=\lim _{q \rightarrow 0} \frac{\psi+(q)}{q^{2}}=\frac{D^{2}}{60 \pi^{2}} \int_{0}^{\infty} d k k^{2} P_{L}(k)$.

Then, for large $k$ (larger than the wavenumber at which the spectral index passes through $n=-3$ ),

$\Delta^{2}(k, D)=\frac{e^{-1 /\left(12 \sigma^{2}\right)}}{(12 \pi)^{1 / 2 \sigma^{3}}}$

which is the same as equation (37), with $\sigma$ in place of $D$.

\section{REDSHIFT-SPACE DISTORTIONS}

In Section 2 we considered some of the properties of the density field evolving in the Zel'dovich approximation. Here we turn our attention to the properties of such a density field when viewed in redshift space. We assume the Zel'dovich approximation throughout this section; in Section 5 we will test the validity of the approximation with $N$-body simulations.

\subsection{Zel'dovich approximation}

In redshift space, the observed radial position of a galaxy is given by its radial velocity, which measures not only the uniform Hubble flow, but also the peculiar motion of the galaxy. Thus the position s of a galaxy in redshift space appears shifted from its true position $x$ by its radial peculiar velocity ${ }^{2}$.

$s=x+[\dot{x} \cdot v(x)] \hat{x}$.

In the Zel'dovich approximation, the peculiar velocity $v$ is related to the displacement $\xi$ at all times by the lineat relation $v[x(q), t]=\xi(q, t)=\beta \xi(q, t)$, where $\beta$, equation (2), is the dimensionless linear growth rate. As discussed in the introduction, we assume an unbiased density field, $b=1$. The position s of a galaxy in redshift space then appears displaced from its initial Lagrangian position $q$ (this initial position $q$ is the same in both real and redshift space):

$s=q+\xi^{s}(q)$

this displacement is given by a redshift displacement $\xi^{3}(q)$ which in the Zel'dovich approximation is

$\xi^{s}(q)=\boldsymbol{\xi}(q)+\beta[\dot{x} \cdot \xi(q)] \hat{x}$

Equation (42) shows that the displacement $\xi^{s}$ of a galaxy in sedshift space is stretched by a factor $(1+\beta)$ along the radial direction. The overdensity $\delta^{s}$ in redshift space is then (compare equation 5)

$\delta^{3}(s)=\int \mathrm{d}^{3} q \delta_{\mathrm{D}}\left[s-q-\xi^{3}(q)\right]-1$.

The radial character of the redshift distortion $\beta(\hat{x} \cdot \xi) \dot{x}$ complicates evaluation of the Fourier transform of the overdensity. However, matters simplify if the structure being observed is far away from the observer, so that the distortions are effectively plane-paraliel. In the plane-parallel approximation, the redshift displacement $\xi^{s}$ becomes $\xi^{5}(q)=\xi(q)+\beta[\xi \cdot \xi(q)] \xi$

where the line-of-sight direction $\hat{z}$ is taken to be fixed. The Fourier transform of the redshift density, equation (43), is then

$\delta^{s}(k)=\int d^{3} q e^{i k \cdot q}\left(e^{i k-t^{\prime}}-1\right)$.

It is convenient to introduce the vector $\boldsymbol{K}$, which is the wavevector $\boldsymbol{k}$ stretched by $(1+\beta)$ along the $z$-axis:

$K=k+\beta(\xi \cdot k) \xi$.

whose magnitude is

$K=k\left(1+2 \beta_{\mu k}^{2}+\beta^{2} \mu_{k}^{2}\right)^{1 / 2}$

with $\mu_{k}$ the cosine of the angle between the wavevector and the line of sight

$\mu_{k}=\hat{z} \cdot \hat{k}$.

In terms of the veclor $K$, equation (45) can be rewritten

$\delta^{s}(k)=\int d^{3} q e^{i k \cdot q}\left(e^{i x \cdot k}-1\right)$.

Just as the displacement $\xi^{3}$ of a galaxy in redshift space is stretched by a factor $(1+\beta)$ along the line-of-sight axis $\xi$, equation

\footnotetext{
${ }^{2}$ From here on we work in distance units of the velocity, where the Hubble parameter $H_{0}=1$.
} 
(44), so also is the displacement conelation function $\psi_{i j}^{s}=\left\langle\xi_{i}^{s} \xi_{j}^{s}\right\rangle$ in redshift space stretched by $(1+\beta)$ factors along each $z$-component of the tensor:

$\psi_{i j}^{s}=\left(\delta_{i k}+\beta \Sigma_{i} \bar{z}_{k}\right) \psi_{k l}\left(\delta_{j l}+\beta \Sigma_{j} \vdots_{i}\right)$.

If the unredshifted displacement field $\xi$ is Gaussian (as is true for an initially Gaussian field evolved in the Zel dovich approximation, Section 2.2), then the redshified displacement field $\xi^{s}$ is also Gaussian.

The derivation of expression (16) for the evolved power spectoum in Section 2.2 did not assume isotropy, and remains valid in redshift space. Thus the redshift power spectrum $P^{\prime}(k)$ is given by equation (16) with the displacement correlation function taken to be the redshift-space quantity $\psi_{i j}^{s}$ :

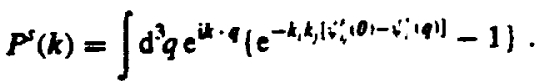

As in equation (16), the unit term in the integrand can be dropped for $k \neq 0$, although again it helps in numerical integrations to retain the term when $k$ is small. Simmetry about the line of sight $z$ implies that the redshift power $P^{\prime}\left(k, \mu_{k}\right)$ is a function of the magnitude $k$ of the wavevector $k$ and of the cosine $\mu_{k}$ of the angle between the wavevector and the line of sight.

The relation (50) between the redshified and unredshifted displacement correlation functions $\psi_{i j}^{j}$ and $\psi_{i j}$ allows the combination $k_{i} k_{j} \psi_{i j}^{s}$ in the integrand of equation (51) to be written conveniently in terms of the vector $K$, equation (46), as $k_{i} k_{j} \psi_{i j}^{s}=K_{i} K_{j} \psi_{i j}$. Then equation ( 51$)$ for the redshift power spectrum becomes

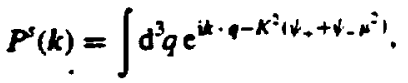

where now $\mu=\hat{K} \cdot \hat{q}$, and $\psi_{x}(q)$ are the (unredshifted) quantities given by equations (21) and (22). Equation (52) is the redshift-space counterpart of equation (24). Integrating equation (52) over the azimuthal angle of $q$ about the vector $K$ yields a Bessel function $J_{0}$ :

$P^{\prime}(k)=\int 2 \pi q^{2} d q d \mu e^{i k q x \mu-K^{2}\left(\psi_{-}+\psi_{-} \mu^{2}\right)} J_{0}\left(k g s \sqrt{1-\mu^{2}}\right)$.

where $s$ and $c$ are

$s=\frac{\beta \mu_{k}\left(1-\mu_{k}^{2}\right)^{1 / 2}}{\left(1+2 \beta \mu_{k}^{2}+\beta^{2} \mu_{k}^{2}\right)^{1 / 2}}$.

$c=\left(1-s^{2}\right)^{1 / 2}=\frac{1+\beta \mu_{k}^{2}}{\left(1+2 \beta \mu_{k}^{2}+\beta^{2} \mu_{k}^{2}\right)^{1 / 2}}$.

Except for the interesting case $s=0$, pursued further in Section 3.4, equation (53) cannot be reduced further to any simple analytic form. Moreover, the oscillatory character of the integrand makes it difficult to evaluate numerically. Further details on the evaluation of the integral (53) are given in Appendix A. In Section 3.5 we adopl a different approach to evaluating the redshifi power spectrum, which is to smooth it.

\subsection{Redshift power spectra for initial power laws}

We now give analytic solutions for the evolution of the redshift power spectrum in the Zel'dovich approximation for initially power-law spectra $P_{L} \propto k^{n}$, for the cases $n=-1$ and $n=-3$.

In Section 2.4 we saw that the $n=-1$ spectrum gave rise to an incoherent random Gaussian velocity dispersion, resulting in a simple damping term for the non-linear power in the Zel'dovich approximation. Extending this to the redshift domain, we can see that the effect will be an additional damping term in the line-of- sight direction - an approximation previously suggested by Peacock (1992) to model the effects of virialized clusters. With the normalization discussed in Section 2.4, we find that the redshift power spectrum is

$\Delta^{s 2}(k, D)=3 \nu D^{2} k^{2}\left(1+\beta \mu_{k}^{2}\right)^{2} \mathrm{e}^{-D^{2} k^{2}}$.

This is the same as the Peacock smoothing with $\sigma_{2}^{2}=D^{2}\left(2 \beta+\beta^{2}\right)$, where $\sigma_{v}$ is the one-dimensional one-point velocity dispersion. Notice that we also recover the linear Kaiser boost factor, $\left(1+\beta_{\mu}^{2}\right)^{2}$. Hence we conclude that this form has some justification beyond its phenomenological foundations for scales where the spectral index is - -1 , and arises as a special case in the model presented here.

For $n=-2$, there appears to be no analytic solution for the redshift power spectrum, unlike the unredshified case, equation (36).

In the case $n=-3$, we find the analytic solution

$$
\begin{aligned}
\Delta^{s 2}(k, D) & =\frac{1}{(12 \pi)^{1 / 2} D^{3}\left(1+2 \beta \mu_{k}^{2}+\beta^{2} \mu_{k}^{2}\right)^{3 / 2}} \\
& \times \exp \left[-\frac{1+2 \beta \mu_{k}^{2}+3 \beta^{2} \mu_{k}^{2}-2 \beta^{2} \mu_{k}^{4}}{12 D^{2}\left(1+2 \beta \mu_{k}^{2}+\beta^{2} \mu_{k}^{2}\right)^{2}}\right] .
\end{aligned}
$$

which is the redshift counterpart of the unredshifted solution (37).

\subsection{Multipole analysis of the redshift power spectrum}

The effect of redshift distortion on the statistical properties of the density field can be best interpreted by a multipole expansion of the redshifted power spectrum. The anisotropic spectrum can be expanded in Legendre polynomials:

$P^{\prime}(k)=\sum_{\ell=0}^{\infty} P_{l}^{\prime}(k) P_{l}\left(\mu_{k}\right)$.

where $P_{r}\left(\mu_{k}\right)$ is the th Legendre polynomial and $\mu_{k}$ is again the cosine of the angle berween the wavevector $k$ and the line of sight. The multipole moments of the power are given by

$P_{l}^{\prime}(k)=\frac{2 \ell+1}{2} \int_{-1}^{1} d_{\mu_{k}} P^{s}(k) P_{\ell}\left(\mu_{k}\right)$

Hamilton (1992) suggested using the ratios of the multipoles as a way of measuring $\beta$, expanding the correlation function from Kaiser's linear theory. Cole, Fisber \& Weinberg (1994) developed this to study the anisotropy of the power spectrum, where non-linear effects could be avoided by limiting the analysis to small wavenumbers. The distortion parameter, $\beta$, could then be estimated from the ratio of monopole and quadrupole power:

$\frac{P_{2}^{\prime}(k)}{P_{0}^{\prime}(k)}=\frac{\frac{4}{3} \beta+\frac{1}{3} \beta^{2}}{1+\frac{3}{3} \beta+\frac{1}{3} \beta^{2}}$.

In the linear regime each observable mode contribules to an independent estimate of $\boldsymbol{\beta}$.

Non-linear effects modify the quadrupole-to-monopole ratio from the linear value $(60)$. In the following section we derive an approximate expression for this ratio in the Zel'dovich approximation, and in subsequent sections we present numerical results for the ratio in the Zel dovich approximation, in $N$-body simulations, and in observations.

\subsection{An approximation to the quadrupole-to-monopole ratio}

In this section we derive an approximation, equation (64), which 
relates the ratio $P_{2}^{i} / P_{0}^{s}$ of the quadrupole and monopole redshift power spectra to the evolution of the untedshifted power spectrum in the Zel'dovich approximation.

In equation (53) for the redshift power spectrum, notice that the Bessel function $J_{0}\left(k g s \sqrt{1-\mu^{2}}\right)$ in the integrand equals one whenever $s$ is zero, which happens in the limit $\beta \rightarrow 0$ (equation 54) and also in the case $\mu_{k}=0$ or 1 where the wavevector is perpendicular or parallel to the line of sight. Moreover in the non-linear limit where $k \rightarrow \infty$, the $K^{2}$ terms in the exponential cause the integrand of equation (53) to decay rapidly as $q$ increases and again the Bessel function is sensibly equal to one (as is also the factor $e^{i k q \mu}$ ) wherever the integrand is non-negligible. In all these cases, the redshified power, equation (53), reduces to the same form as its unredshifted counterpart, equation (24), but with $\psi$, in the latter replaced by $(K / k)^{2} \psi_{z}$, where $K / k=1+\beta_{\mu_{k}^{2}}^{2}$ (equation 47 ) given that $s=0$ and $c=1$ (equation 55). In other words, the redshifted power equals the unsedshifted power evolved forward by $1+\beta_{\mu k}^{2}$ in the growth factor $D(t)$ :

$P^{\prime}(k, D) \sim P\left[k,\left(1+B \mu_{k}^{2}\right) D\right]$.

As it happens, this same equation (61) is also valid in the linear regime, according to the usual linear relations $P(k, D)=D^{2} P_{1}(k)$ and $P^{\prime}(k, D)=\left(1+\beta \mu_{k}^{2}\right)^{2} P(k, D)$. Thus approximation (61) is valid (i) in the limit $\beta \rightarrow 0$, or (ii) if $\mu_{k}=0$, i.e. if the wavevector $k$ is perpendicular to the line of sight, or (iii) if $\mu_{k}=1$, i.e. if the wavevector $k$ is parallel to the line of sight, or (iv) in the highly nonlinear regime, or $(v)$ in the linear regime. Evidently the approxima. tion is of some generality.

Let us consider more closely the limiting case $\beta \rightarrow 0$, for which the approximation (61) becomes exact (within the contex of the Zel'dovich approximation). Expanding equation (61) as a Taylor series for small $\beta$ gives

$P^{s}(k, D)=P(k, D)+\beta \mu_{k}^{2} D \frac{\partial P(k, D)}{\partial D} \quad(\beta \rightarrow 0)$.

Here the redshift power spectrum is the sum of a constant term and a term proportional to $\mu_{k}^{2}$. which implies that the redshift power spectrum is a sum of monopole and quadrupole terms, for small $\beta$. The ratio of quadrupole and monopole powers for small $\beta$ is, from equation (62),

$\frac{P_{2}^{\prime}(k)}{P_{0}^{j}(k)}=\frac{4 \beta}{3} \frac{\partial \ln P(k, D)}{\partial \ln \left(D^{2}\right)} \quad(\beta \rightarrow 0)$.

Amongst other things, equation (63) predicts that the quadrupole redshifted power $P_{2}^{\prime}(k)$ goes through zero where the unredshified power $P[k, D(t)]$ reaches its maximum as a function of time in the Zel'dovich approximation.

We Dow propose a generalization of the result (63) to arbitrary $\beta$, which is consistent with and to some extent motivated by approximation (61), and wbich accords with the numerical results of Section 3.6 below, which are further supported by the $N$-body simulations in Section 4.1. The numerical results indicate that, at least in the case of initially power-law power spectra, the quadrupole-to-monopole ratio $P_{2}^{\prime} / P_{0}^{\prime}$ appears to satisfy a simple scaling law with $\beta$, such that the shape of the ratio is insensitive to $\beta$, while its amplitude is proportional to the usual linear ratio (strictly, the Dumerical results are for smoothed power spectra, but we assume that the results bold also without smoothing). We further find that the zero-crossing of the quadrupole scales with $\beta$ such that $D^{2}-1 /(1+\beta)$. These empirical sesults, combined with equation
(63), suggest the general approximation

$$
\frac{P_{2}^{\prime}(k)}{P_{0}^{5}(k)}=\frac{\frac{4}{3} \beta+\frac{4}{3} \beta^{2}}{1+\frac{3}{3} \beta+\frac{1}{3} \beta^{2}} \frac{\partial \ln P\left[k_{1}(1+\beta)^{1 / 2} D\right]}{\partial \ln \left(D^{2}\right)} \text {. }
$$

The approximation (64) relates the quadrupole-to-monopole ratio $P_{2}^{\prime} / P_{0}^{t}$ of the redshifted power spectrum to the evolution $\partial \ln P / \partial \ln D^{2}$ of the unredshified power spectrum in the Zel'dovich approximation. Although we have numerical support for the approximation (64) only in the case of initially power-law spectra, we suggest that it is likely to be a good approximation for arbitrary power spectra.

\subsection{The generating function of smoothed power spectrum multipoles}

So far we have obtained a number of analytic results for the redshift power spectrum in the Zel'dovich approximation, but in general it is necessary to resort to numerics. Unforunately, numerical integra. tion of the redshift power spectrum, equation (53), while feasible, is unpleasant (see Appendix A). One way to sidestep the numerical difficulties is to smooth the power spectrum. This is not such a bad idea because it is necessary to measure a smoothed power spectrum from observations. That is, the Fourier modes measured in a catalogue are already convolved with the window function of the catalogue, and one may choose to smooth the power spectrum further to reduce error bars. In addition, smoothing makes defining quantities such as the zero-crossing of the quadrupole, discussed in Section 3.6, more robust to random errors.

The smoothed (unredshified) power spectrum is defined as

$\dot{P}(\bar{k})=\int \mathrm{d}^{3} k P(k) W_{N}(k, \bar{k})$.

We choose to adopt a power law times Gaussian smoothing kernel $w_{N}(k, \bar{k})=\frac{k^{2 N} \exp \left(-k^{2} / \hat{k}^{2}\right)}{2 \pi \bar{k}^{2 N+3} \Gamma[(2 N+3) / 2]}$.

which is analytically convenient, and goes to a delta function in the limit $N \rightarrow x$. In subsequent sections we use $N=1$.

For the redshift power spectrum, the smoothed harmonics can be defined similarly by

$\dot{P}_{l}^{s}(\bar{k})=(2 \hat{k}+1) \int \mathrm{d}^{3} k P^{s}(k) P_{\mathrm{f}}\left(\mu_{k}\right) W_{i}(k, \bar{k})$.

The ratio $\dot{P}_{0}^{j}(\bar{k}) / \dot{P}_{2}^{\prime}(\bar{k})$ of smoothed quadrupole and monopole harmonics satisfies the usual equation $(60)$ in the linear limit.

The whole hierarchy of smoothed spectra, for various $N$ and $\ell$, can be derived from the generating function

$G(a, b)=\int d^{3} k P^{\prime}(k) e^{-a k^{2}-b k^{2} \mu_{i}^{2}}$.

In terms of this generating function, the monopole harmonic of the redshift power spectrum, smoothed with the window $W_{N}^{\prime}$, is

$\dot{P}_{0}^{\prime}(\dot{k})=\left[-\frac{\partial}{\partial a}\right]_{a=k-2}^{v}\left\{\frac{G(a, b=0)}{2 \pi \bar{k}^{2 N+3} \Gamma[(2 N+3) / 2]}\right\}$

while the quadrupole redshifted power smoothed with the same window is

$$
\begin{aligned}
& \dot{P}_{2}^{\prime}(\dot{k})= \\
& \frac{5}{2}\left[\frac{\partial}{\partial a}-3 \frac{\partial}{\partial b}\right]_{b=0}\left[-\frac{\partial}{\partial a}\right]_{a=k^{-2}}^{N-1}\left\{\frac{G(a, b)}{2 \pi k^{2}+3 \Gamma[(2 N+3) / 2]}\right\} .
\end{aligned}
$$




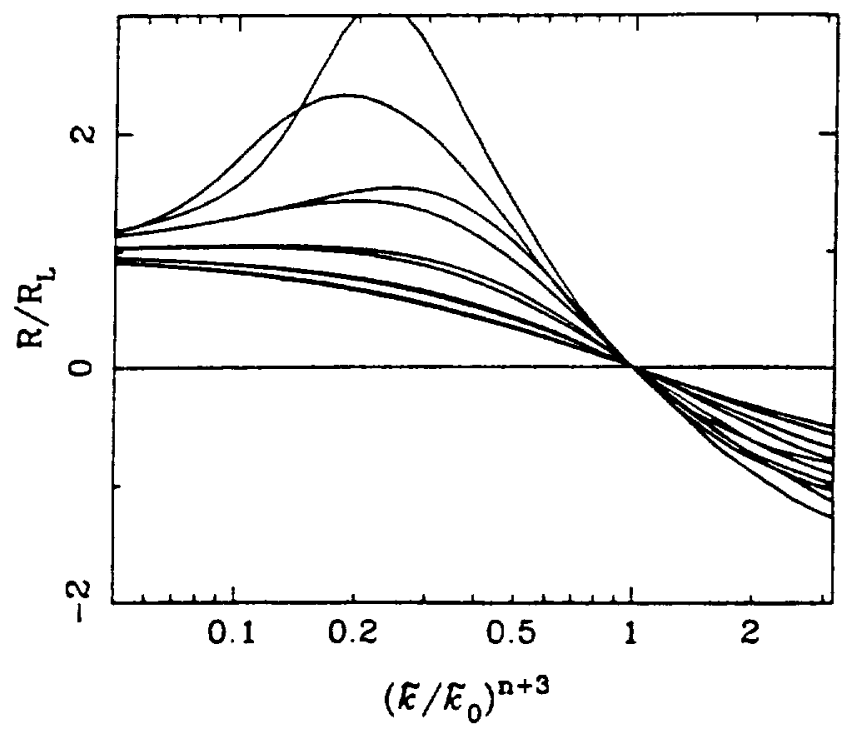

Figure 2. Plots of the ratio $R$ of smoothed quadrupole and monopole powers against $\left(\bar{k} / \bar{k}_{1}\right)^{n-3}$ for initially poucr-law spectra with indices. from lower curve to upper, of $n=-1,-1.5,-2,-2.5$ and -2.9 . In each case cunes for $\beta=0$ and 1 are shown. which lie close to each other (and practically on top of each other for $n=-1$ and -1.5 ) when $R$ is scaled to the linear amplitude $R_{L}$ and the zero-crossing scale $K_{1}$. For each value of the speciral index. $\beta=0$ gives the slightly higher scaled ratio.

Substituting equation (51) for the sedshift power spectrum into the generating function $(6 S)$ gives

$G(a . b)=\int \mathrm{d}^{3} q \mathrm{~d}^{2} k \mathrm{e}^{i k \cdot q-k, k, \psi, \cdots q)}$

where $\Psi_{i j}$ is the matrix

$\Psi_{1 j}(q)=a \delta_{1 j}+b \vdots_{i=1}^{\prime}+\psi_{11}^{\prime s}(0)-\psi_{1 j}^{s}(q)$.

The $k$-part of the integral (71) is the Fourier transform of a Gaussian, which solves in the usual way to give

$G(a . b)=\int \frac{\pi^{3 / 2} d^{3} q}{|\Psi|^{1 / 2}} e^{-q^{i} \psi_{\alpha q}^{-1 / 4}}$

where $|\boldsymbol{\Psi}|$ is the determinant of $\Psi_{\imath j}$,

$$
\begin{gathered}
|\boldsymbol{\Psi}|=\left(\psi_{+}+a\right)\left\{\left(\psi_{+}+\psi_{-}+a\right)\left[\psi_{+}(1+\beta)^{2}+a+b\right]\right. \\
\left.+\mu^{2} \psi_{-}\left[a\left(2 \beta+\beta^{2}\right)-b\right]\right\}
\end{gathered}
$$

with $\mu=\xi \cdot \dot{q}$ and $\psi_{z}(q)$ given by equations (21) and (22), and $\Psi_{q q}^{-1}=\dot{q}_{1} \Psi_{i j}^{-1} \dot{q}_{i}$ is the $q q$-component of the inverse of $\Psi_{i j}$,

$$
\begin{aligned}
\Psi_{4 q}^{-1}= & \frac{\left(\psi_{+}+a\right)}{|\Psi|}\left\{\psi_{+}+a\right. \\
& \left.+\left(1-\mu^{2}\right)\left[\psi_{+}\left(2 \beta+\beta^{2}\right)+b+\mu^{2} \psi_{-} \beta^{2}\right]\right\} .
\end{aligned}
$$

Evaluation of the smoothed monopole and quadrupole spectra from equations (69), (70) and (73) still involves a double integral over $\mu$ and $q$. However, the integrand here is well-behaved (at least for small $N$, such as $N=1$ ) and depends only on elementary functions [besides $\psi_{x}(q)$ ]. This compares with the unsmoothed case (Appen$\operatorname{dix} A$ ), where the integrand is ill-behaved, and evaluating the harmonics involves a double integral over an infinite sum involving special functions.

\subsection{Numerical results for quadrupole and monopole power}

From the generating function of multipole moments, equation (73), we have calculated numerically the smoothed quadrupole and monopole moments of the power spectrum for initially power-law spectra $P_{L} \times k$ with various values of the inde $x n$. and for various $\beta$. The smoothing kernel is 11 . equation (66), with $N=1$. Fig. 2 show's the ratio $R$ of the smoothed quadrupole and monopole powers

$R=\frac{\dot{P}_{:}^{\prime}(\dot{k})}{\bar{P}_{0}^{\prime}(\bar{k})}$

for a representative sample of cases. $n=-1 .-1.5,-2,-2.5$ and -2.9 , and for $\beta=0$ and 1 in each case. Values intermediate between these give cunes intermediate to those plotted. The plotted ratio has been divided by the linear ratio, $R_{L}=\left(\frac{4}{3} \beta+\frac{1}{5} \beta^{2}\right) /\left(1+\frac{3}{3} \beta+\frac{1}{5} \beta^{2}\right)$, equation (60), and the wave. number has been scaled to the zero-crossing scale. $\bar{k}_{0}$

It is immediately appatent from Fig. 2 that the shape of the quadrupole-to-monopole ratio $R$ depends on the spectral index $n$. but is insensitive to $\beta$ at fixed $n$, at least for $n \geq-2.5$. The insensitivity to $\beta$ is an interesting result. and we have previously invoked this insensitivity in proposing the approximation (64) to the (unsmoothed) ratio. As to the spectral index. Fig. 2 shou's that, for $n \leqslant-2$, the ratio $R$ aclually rises up above its linear value before turning over and going through zero. Physically. the linear squash. ing effect is first enhanced by caustics in redshift space as structures approach tumaround. and is then suhsequently negated by fingers. of-God as structures collapse. Fig. 2 indicates that for $n \leq-2$ the caustic effect wins over the finger-of-God effect at translinear scales. However. as the cnhancement peak scales as $\dot{k}_{F_{k}}-0.2^{1, n+\xi_{i}} \dot{k}_{i}$. we find that $R$ decreases for $n \leq-2$. for fixed $\beta$. in the regime $k-k_{0}$.

A useful filling function to the curves in Fig. 2 is

$R=R_{L} e^{-1 n+1 i^{2}}\left[1-\left(\dot{k}_{i} \bar{k}_{\mathrm{i}}\right)^{0 \mathrm{n}+n+3)}\right]$.

which is accurate to within 10 per cent over the range $0.1<\bar{k} / \bar{k}_{\mathrm{u}}<1$. and $-\overline{3}<n<-1$. We shall use the fitting function (77) in filting the obseried ratio $R$ measured in the QDOT plus 1.2Jy surey to $\beta$ and $n$, in Section 4.2.

The zero-crossing scale of the quadrupole power also contains useful information about $\beta$ and the speciral index. The zero-crossing scalc discussed below refers strictly to that of the smoothed quadrupole power. but the zero-crossing scale of the unsmoothed quadrupole is not greally different.

Suppose that the zero-crossing of the smoothed quadrupole power $\dot{P}_{2}^{\prime}(\dot{k})$ is obsened to occur at navevector $\dot{k}_{0}$. Define a corresponding real scale by $\dot{q}_{0}=\alpha / \bar{k}_{0}$. Where $\alpha$ is a fitting constant. discussed below. Then we find that the dimensionless amplitude of the displacement correlation function at the zero-crossing scale $\dot{q}_{u}$ is fitted tolerably well by the following fitting formula:

$\frac{\dot{\nu}\left(\dot{g}_{1}\right)}{\dot{q}_{0}}=\frac{C}{1+\beta} . \quad \dot{g}_{11}=\alpha / \dot{k}_{v}$.

In the Zel'dovich approximation, formula (78) $w$.jth the values (we adopt here the value of $\alpha$ that best fits the $\alpha^{\prime}$-body simulations reported in Section 4.1, since the same value uorks well also for Zel'dovich)

$\alpha=2.3, C=0.19$

is accuraie to better than 20 per cent for initially power-law spectra over the ohsenationally inieresting range of spectral indexes $-2 \leq n \leq-1$ and linear growth rate parameter $0 \leq \beta \leq 1$. As a matter of interest, in the case $n=-1$ the exact analytic result (in the Zel'dovich approximution) is $\psi_{-}\left(\bar{g}_{11}\right) / \dot{q}_{i j}^{i}=1 /\left[\alpha^{2}(1+\beta)\right]$. Physically, it is not 100 surprising that the zero-crossing of the quadrupole should occur when the ims displacement $\psi_{-}^{1 / 2}(q)$ between pairs has reached an appreciable fraction of their separation $q$. 
The amplitude of the power spectrum itself at the zero-crossing $\dot{k}_{u}$ is a more complicated function of spectral index. During linear evolution. the dimensionless smoothed monopole redshifted power $\bar{\Delta}_{v}^{s-i}(\bar{k})$ is related to the dimensionless displacement correlation function $\psi_{-}(\bar{q}) / \bar{q}^{2}$ at $\dot{q}=\alpha / \bar{k}$ by (compare equation 32: note that the factor $\Gamma[(5+n) / 2] / \Gamma(5 / 2)$ below comes from smoothing)

$$
\begin{aligned}
\dot{\Delta}_{v}^{s}(\dot{k})= & \left(1+\frac{2}{3} \beta+\frac{1}{5} \beta^{2}\right) \frac{2}{\pi}(2-n) \sin [(-n-1) \pi / 2] \Gamma(1-n) \\
& \times \frac{\Gamma[(5+n) / 2]}{\Gamma(5 / 2)} \alpha^{3+n} \frac{\psi+(\alpha / \bar{k})}{(\alpha / \bar{k})^{2}} .
\end{aligned}
$$

Non-linear evolution, which is starting to become important at zerocrossing. will modify the power from the linearly evolved value (80). Selting aside this effect of non-linearity, one concludes from equation ( 80 ). combined with the approximation (78). that the amplitude of the monopole power at zero-crossing is sensitive to the spectral index $n$. but relatively insensitive to $\beta$ [the $1 / 1]+\beta$ ) factor in equation (78) tending to cancel the $1+\frac{\beta}{3} \beta+\frac{1}{5} \beta^{2}$ factor in equation $(80)$ ]

It is worth emphasizing this interesting if somewhat disappointing result: one might have hoped that the amplitude of the observed monopole power at the zero-crossing of the quadrupole would provide a measure of $\beta$ in the mild!y non-linear regime, offering a check on the value of $\beta$ measured from the quadrupole-10-monopole ratio in the linear regime, and perhaps a test of the bias $b$. However, the hope is not realized: the power at zero-crossing is sensitive mainly to the spectral index, not to $\beta$.

\section{COMPARISON WITH SIMLLATIONSAND OBSERVATIONS}

\subsection{Comparison with simulations}

Given the approximate nature of the Zel'dowich mapping assumed in Sections 2 and 3 . it is importunt to check the accuracy of these results with $\Lambda$-bod! simulations. where non-linear eftects can be accounted for on smaller scales. The simulations that we ran used the $\mathrm{AP}^{3} \mathrm{M}$ code of Couchman (1991). generalized to low $\Omega$ by Peacock \& Dodds (1994). on a $64^{3}$ grid with $64^{3}$ particles.

We ran simulations with power-law initial spectra with indices $n=-1,-1.5$ and -2 , both for $\Omega=1$. and for a low- $\$$ spatially flat model with $\Omega=0.3$ and a vacuum-energy contribution of $\Omega_{.}=0.7$, corresponding to $\beta=0.5$. In each case three simulations were computed with diftering random Gaussian initial conditions, 18 simulations altogether. We assumed no bias in the density field, and evolved all the models for 300 time-steps so as to erase the effects of using the Zel'dorich approximation in the initial setting up of the simulation.

Power multipoles were calculated by assigning densities to the grid by the cloud-in-cell algorithm and fast Fourier transforming the cube. The shot-noise component of the power was subtracted and the resulting Fourier space compressed by averaging over azimuthal angles. The multipoles were then calculated by a leastsquares fit to each mode:

$x^{2}(k)=\sum_{i} u_{i}(k)\left[j^{2}\left(k, \mu_{1}\right)-\sum_{t} \dot{\Delta}_{i}^{2}(k) \mathcal{P}_{i}\left(\mu_{1}\right)\right]^{2}$.

The weighting scheme used was the inverse variance. $n_{i}(k)=1 / \sigma^{2}\left(k, \mu_{i}\right)$. calculated from the intrinsic cosmic variance. $\sigma(k)=\sqrt{2} s^{2}(k)$. for each mode. The resulting power multipoles were then smoothed using the kernel function $W_{i}$ with $\Lambda=1$, equation (66). and an inverse variance weighting scheme.

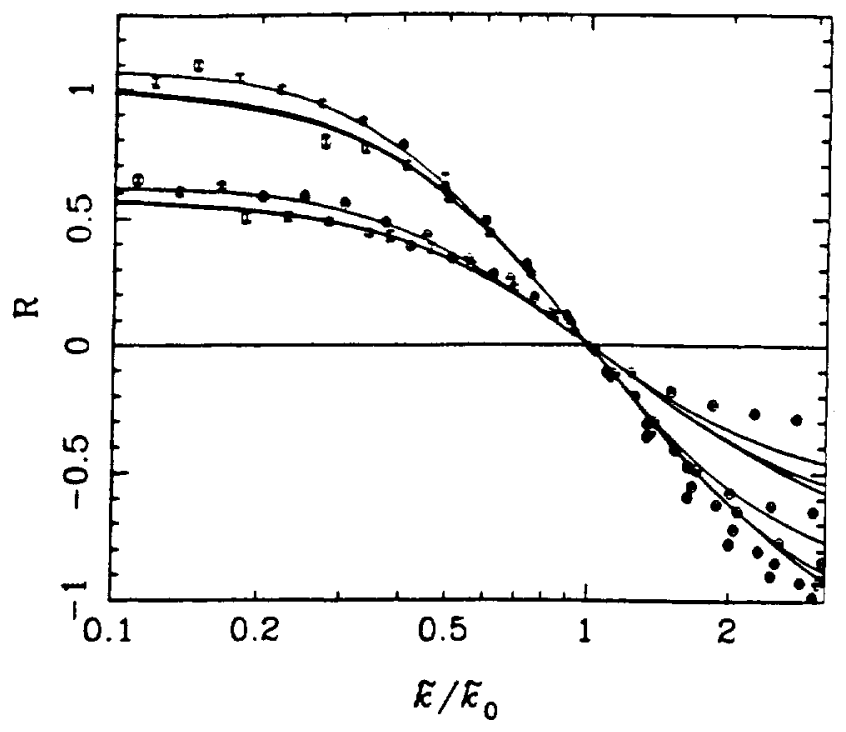

Figure 3. Plots of the ratio $R$ of smoothed monopole and quadrupols powers scaled by the zero-crossing. $\dot{k}_{0}$. From top to bottom or scales $\bar{k}<\dot{k}_{j}$ are plotted the numerical results for the Zeldovich evolution for each power spectrum (solid lines). for $\beta=1$ with $n=-1,-1.5$ and -2 (top three lines) and $\beta=0.5$ with $n=-1,-1.5$ and -2 (bottom three lines). Overlaid ate the results from a sel of $N$-body simulations with the same initial parameters (points). Errors on the simulations are from cosmic variance.

Generally, we found that the Zel dovich approximation ceased to provide a good approximation to the evolution of monopole or quadrupole power at moderately non-linear epochs. This is to be expected. since. as discussed in Section 2.3, the expansion of structures following collapse which occurs in the $\mathrm{Zel}$ dovich approximation causes power to reach a maximum and then decline, whereas in reality power continues to increase monotonically:

However, as shown in Fig. $\mathbf{3}$, the Zel'dorich approximation does appear to reproduce remarkably well the amplitude and shape of the ratio $R$ of the quadrupole and monopole powers seen in the $\Lambda$-body. simulations, for scales down to the zero-crossing of the quadrupole, $\bar{k} \leq \bar{k}_{i j}$. Small deviations occur at small $\dot{k}$, but these can be attributed 10 the lack of contribution from smaller wavenumbers in the smnothing process of the simulations. Small deviations are also seen in the $n=-2$ spectra, at least some of which may arise from cosmic variance and the influence of the finite box size on the longest wavelengths in the simulations. However. the change in shape of the quadrupole-to-monopole tatio with spectral index predicled by the Zel dovich approximation is clearly seen in the simulations. All the models fail in the regime $k>k_{u}$. where we expect virialized clusters to provide the strongest redshift distortions (Jackson 1972).

The scaling relation suggested by equation (7S) for the amplitude of the displacement covariance at the zero-crossing of the quadrupole is also found to hold for the simulations. However. the amplitude $C$ predicted by the Zel dovich approximation was not so accurate. Applying a least-squares fit of the model to the ensemble of 15 simulations, we found

$\alpha=2.3 \pm 0.4, \quad C=0.54 \pm 0.07$

with a formal $\lambda^{2}=15.6$ for 16 degrees of freedom. In general, while the form of the scaling relation (7S) holds. the Zel dorich approximation underestimates the wavenumber at zero-crossing of the quadrupole by a factor of approximately 2 in the cases considered. This can be understood as arising from the outflow of particles which follows caustic formation in the Zel dovich 


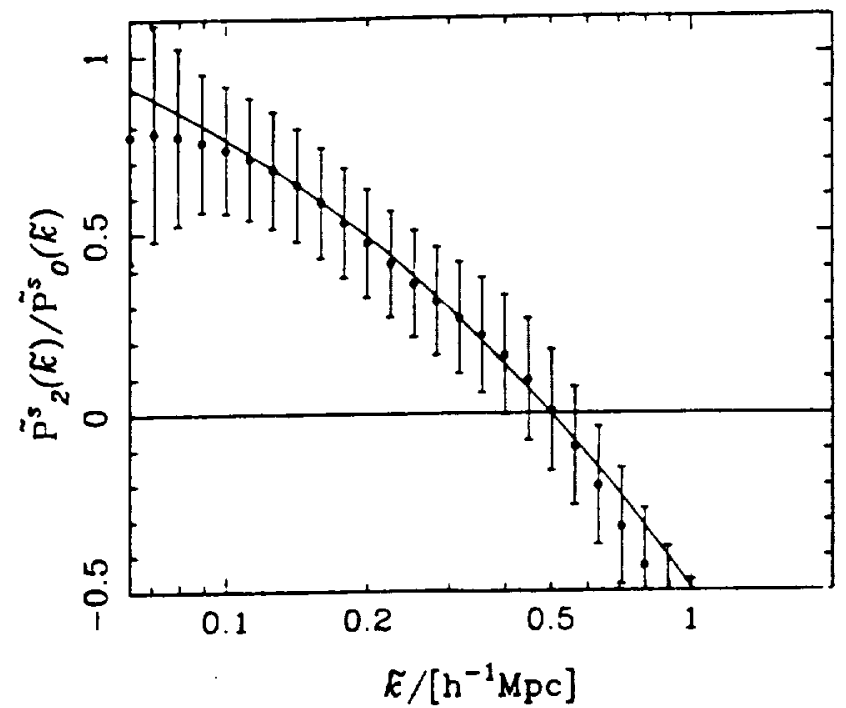

Figure 4. Plut of the ratio $R$ of smosihed monopole and quadrupole poucers as a funcrion of wasenumber $k$ for the nerged QDOT plus 1.2.J! redshift surey. The solid line is a fil to a model $w$ ith $\beta=0$. and $n=-2.5$. und $w$ ith

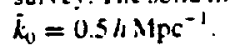

approximation. This ourflow' exaggerales the 'finger-of-God' effect, causing the zero-crossing of the quadrupole to appear at larger scales in the Zel dovich approximation than is actually the case.

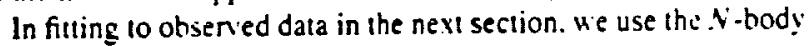
simulations to calibrate the zero-crossing of the quadrupole.

\subsection{Application to the QDOT plus 1.2.Jy redshift survey}

We have applied the results obiained above to estimate the distortion parameter. $\beta$. and the local prinordial spectral index. $" 1$. from the amplitude and shape of the ratio $R$ of smoothed quadrupule and monopole powers observed in the merged QDOT plus 1.2-Jy IRAS redshift survey. We further infer the variance $\sigma_{s}^{2}$ of counts in $8 h^{-1} \mathrm{Mpc}$ spheres from the obseried scale of the zero-crossing of the quadrupole. The observed quadrupole-to-monopole ratio, taken from Hamilton (199.5). is shown in Fig. 4. Superimposed on the observations is a model with $\beta=0.8$ and $n=-2.5$. The zerocrossing is estimated to be at $\bar{k}_{n}=0.5 \pm 0.1 \mathrm{~h} \mathrm{Mpc}^{-1}$.

We usc the fitting function (77) to fit the amplitude. which depends on $\beta$. and the shape, which depends on $n$. of the observed quadrupole-to-monopole ratio $R$. We construct a likelihood function

$\mathcal{L}=\exp \left(-a_{1} M_{i j} a_{!}\right)$

where $a_{i}=$ (data - model) is the difference betueen the ith data point and the model prediction. $M_{i j}$ is the data covariance matrix uhose diagonal terms comprise the uncertainty on each data point and whose off-diagonal terms are estimated assuming that the underlying data have independent Gaussian-distributed errors, and the major source of correlations comes from smoothing. As out model breaks dou'n on scales smaller than the zero-crossing point, and the errors on the observed quadrupole diverge at low wavenumber. he restrict our analysis to wavemodes in the range $0.06<\dot{k} /\left(h \mathrm{Mpc}^{-1}\right)<0.5$.

Fig. 5 show's the marginalized likelihood in the in. $\beta$; plane. As $n \rightarrow-3, \beta$ is forced to infinity by our fitting function. The like. lihond function selects this range as the better fit to the data, owing to the slight inflection in the quadrupole-10-monopolc ratio around

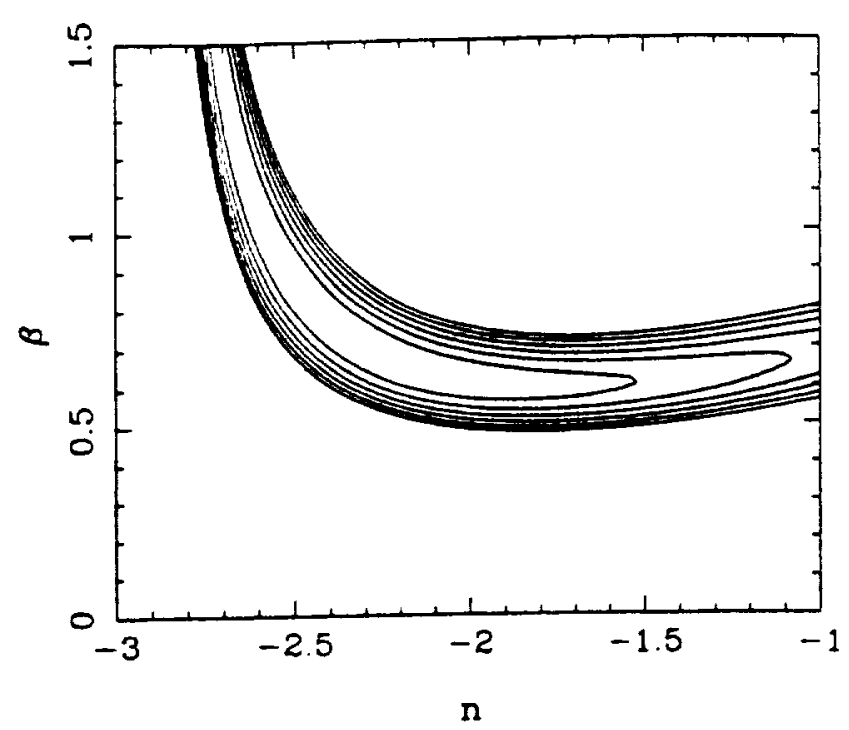

Figure 5 . Likelihoud function $\mathcal{L}(\beta, n \mid \mathrm{d} a t 3)$. of $\beta$ and the loxal specral index. $n$. from the quadrupole-to-monopole ratio of the merged QDOT plus 1.2-Jy redshift suney. Contours are spaced by oln $\mathcal{C}=-0.5$ with the inner contour delineating the $6 S$ per cent and outer contour the 95 per cent confidence segion. While the spectral inde $x$ is not strongly constrained. $\beta<0 . i^{2}$ is ruled out at the 95 per cont level. This rules nut $\Omega<0.3$ if $I R+S$ galuxies are unbiased.

$\bar{k}=0.2 h \mathrm{Mpc}^{-1}$. However, if we consider only the 95 per cent confidence limit given by the outer contour, low values of $\beta<0.5$ are strongly excluded. In terms of the galaxy bias parameter, this implies that the density parameter $\Omega>0.3 b^{5 / 3}$. The value of the primordial spectral index on these scales is not strongly constrained. but is consistent with $n=-2$.

We estimatc the clustcring amplitude $\sigma_{\S}$ from the scale of the zero-crossing of the quadrupole, using equation (78) with the paramcters ( 82 ) calibiated from the $N$-body simulations. In Fig 6 we plor the marginalized likelihood function $\Omega\left(\sigma_{R}\right)=\int d_{N} \mathrm{~d} d n$ $\mathcal{L}\left(\beta . n . \sigma_{s} /\right.$ data). We deduce that $\sigma_{s}=0.7=0.05$ where we quote 95 per cent errors. This is in very good agreement with values found by other methods for the IRtS galaxy catalogues (e.g.. Fisher et al. 1994: Heavens \& Taylor 1995) if $I R A S$ galaxies are unbiased.

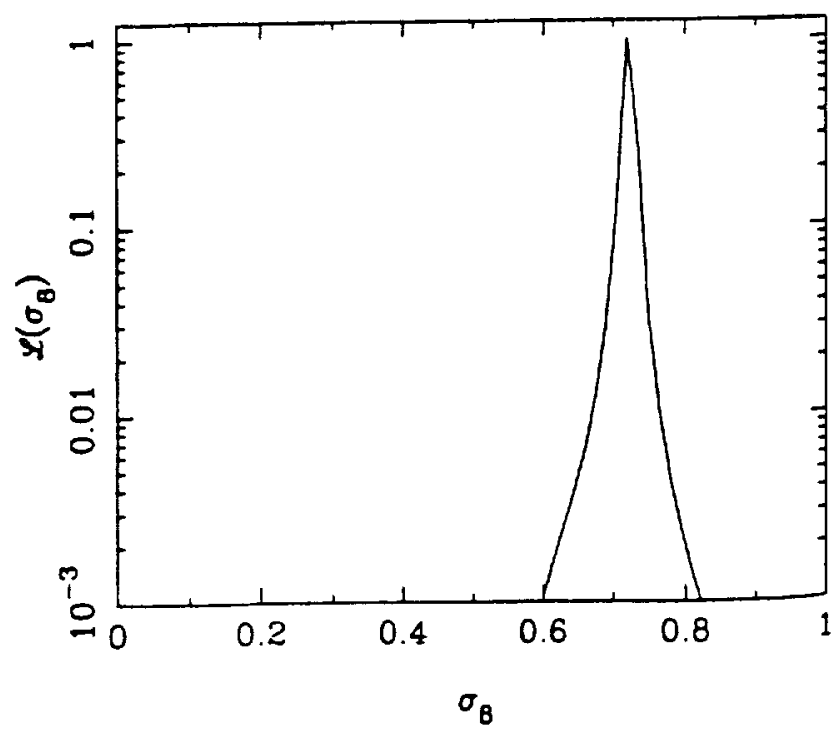

Figure 6. Marginalized likclihond function $\int(0,1)$ f the variance in $s h^{-1} \mathrm{Mps}$ spheres infered from the zero-crossing of the QDOT plus 1.2-J: quadrupole. 
Given the indirce method of our measurement, we find this very encouraging.

\section{SUMMARY}

The main aim of this paper hus been to study the redshift distortion of the power spectrum in the moderately non-linear reginte. Our approach is motivated by the consideration that structures may appear in redshift space to be more non-linear than they really are for example. regions that are just turning around in real space appear as caustics in redshift space. This leads us first to work in Lagrangian space, and secondly to use the Zel dovich approximation. which is in effect linear theory expressed in Lagrangian space.

We started by deriving an expression relating the power spectrum to the Lagrangian displacement field. We used this to determine the evolution of the power spectrum in the Zel dovich approximation, first in real space, then in redshift space in the plane-parallel approximation. We presented some analytic solutions for initially power-litw spectra. In particular. we showed that a spectrum with index $n=-1$ gives rise to an incoherent Gaussian displacement field, producing non-linear redshift distortions with the same form as the Kaiser-Peacock model.

W'e derived various analytic and numerical results for the observationslly interesting ratio $R$ of quadrupole and monopole redshifi powers, whose value in the linear regime, $R_{L}=\left(\frac{1}{\xi} \beta+\frac{1}{3} \beta^{2}\right) /\left(1+\frac{2}{3} \beta+\frac{1}{3} \beta^{2}\right)$. yields a measure of the distortion parameter $\beta$. In the Zel'dovich approximation, the amplitude of the ratio $R$ is set by the linear value $R_{L}$. but the shaps of $R$ as a function of wavenumber $k$ depends mainly on the spectral index $n$, and is insensitive to $\beta$ at fixed $n$. The zero-crossing of the quadrupole power occurs at the point where the Zeldovich power spectrum is a maximum as a function of time.

We have tested the Zel dovich results against $N$-body simulations with initiall! power-law spectra, in both $\Omega=1$ and low- $\Omega$ models. The simulations show that the Zel'dovich approximation ceases to provide a good approximation to the power spectrum at moderatcly non-linear epochs. Remarkably, however. the Zel'dovich approximation predicts rather well the amplitude and shape of the quadrupole-10-nonopole ratio $R$ on scales down to the zerocrossing of the quadrupule. when $R$ is scaled to the seale of the zerocrossing. The Zel'dovich approximation underestimates the wavenumber at zero-crussing by a factor of about 2 , although it predicts correctly the way in which the zero-crossing scales with $\beta$ and $n$.

We have applied these findings to estimate the distortion parameter $\beta$, the local spectral index $n$. and the variance $\sigma_{i}^{2}$ of counts in $8 h^{-1} \mathrm{Mpc}$ spheres. from the quadrupole-to-monupole ratio $R$ measured in the merged QDOT plus 1.2-Jy redshift survey. We find that the distortion parameter is constrained to $\beta>0.5$ at the 95 per cent level. The spectral index is not well constrained, but is consistent with $n=-2$ at translinear scales. The clustering amplitude $\sigma_{8}$. inferred from the scale of the zero-crossing of $R$, is $\sigma_{8}=0.7 \pm 0.05$, consistent with other estimates.

The success of the $Z$ el dovich model in describing the quadrupole-to-monopule ratio $R$ suggests that departures from the linear value $R_{L}$ at translinear scales are caused mainly by infall on to clusters, not by virialized cluster cores

\section{ACKNOWLEDGMENTS}

ANT is supported by a PPARC research assistantship, and thanks Alan Heavens and John Peacock for stimulating discussion. AJSH thanks George Efstathiou for the hospitality of the Nuclear and Astrophysics Laboratory at Oxford University, whire AJSH enjoyed the support of a PPARC Visiting Fellowship during 1994/5, and he thanks John Peacock for hospitality during a visit to ROE. where this collaboration began. AJSH appreciates support from NSF grant AST93-19977. and NASA Astrophysical Theory Grant NAG 5-2797. While in the process of completing this work we became aware of a similar paper by Fisher \& Nusser (1996) who reach similar conclusions.

\section{REFERENCES}

Abramouritz M.. Stegun 1.A., 1968, Handbook of Mathematical Functions. Dover, New York

Ballinger W.E., Heavens A.F.. Taylor A.N.. 1995, MNRAS, 276, L59

Bond J.R.. Couchman H.M.P., 1987, preprint

Cole S.. Fisher K.B., Weinberg D.H.. 1994, MNRAS, 267.785

Couchman H.M.P.. 1991, ApJ. 36S, L23

Efsiathiou G., 1990. in Peacock J.A.. Heavens A.F.. Davies A., eds. Proc. 36th Scotrish Universities Summer School in Physics. Physies of the Early Liniverse. Adam Hilger. Bristol

Fisher K. B.. Nusser A.. 1996. MI.NRAS. 279, L1

Fisher K.B.. Sitharf C.A.. Labav O.. 1994. MNRAS. 266. 219

Fisher K.B. Lahav O.. Huftman Y.. Lynden-Bell D., Zaroubi S., 1995, MNRAS, 272. 8S:

Gorski K..11. 19SS. ApJ. 332. L7

Hamilton A.J.S., 1992. ApJ, 385. LS

Humilton A.J.S., 1995. in Proc. XVth Rencontres de Moriond, Clustering in the Universe, in press

Hamilton A.J.S.. Culhane M. 1996. M.NRAS, 278, 73

Hejvens A.. Taylot A.N.. 1995. M.NRAS. 275, 483

Hivon E.. Buuchel F.R.. Colombi S., Juszkiewicz R., 1995, A\&A. 29S, $6+3$

Hui L.. Bertschinger E.. 1995, preprint

Jackson J.C.. 1972. MINRAS, 156, Ip

Kuiser $N$.. 19S7, MNRAS, 227, 1

Munn R.G.. Heavens A.F., Peacock J.A.. 1993, MNRAS. 263. 799

Munin A.S.. Yaghom A.M., 1975. Statistical Fluid Mechunics. Vols $1 \& 2$ MIIT Press. Cambridgre

Peacock J.A.. 1992 in Martinez V.. Porrilla M., Sáez D., eds. Nex Insights into the Liniverse. Proc. Valencia summer schnol. Springer, Berlin, p.1

Peacock J.A.. Dodds S.J., 1994, M.YRAS. 267, 1020

Peehles P.J.E.. 1980. Large Scale Siructure in the Universe. Princelon University Press. Princeton

Schneider P.. Banelmunn M., 1995, MNRAS. 273. 475

Taylur A.N., 1993, in Bouchet F., Lachieze-Rey M., eds. Proc. Cosmic Velocity Fields. 9in laU Conf. Editions Frontières. Gif-sur-Y'velte. p. 585

Zaroubi S.. Hoffman Y'. 1996. ApJ, 462. 25

Zel dorich Y.B.. 1970. A\&.A, 5.84

\section{APPENDIXA}

This section shows how to evaluate the 'difficult integral (53) for the redshift power spectrum in the Zel'dovich appruximation. The integral is a two-dimensional integral over $\mu=\dot{K} \cdot \dot{q}$ and separation $q$. Wic choose to develop the integral over $\mu$ analytically as an infinite sum. both because the numerical integral over $\mu$ is itself unpleasant. and because the expression as an infinite sum allows the $q$-integrand to he concinued analyically into the complex $g$-plane, which stabilizes tha integration or er $q$.

For small kigs, the Bessel function $J_{0}\left(k q s \sqrt{1-\mu^{2}}\right)$ in the integrand can be expunded as a power series in $1-\mu^{2}$. Integration of the leading lirm (which is 1 ) in the series leads to an expression similar to (25), and higher terms in $1-\mu^{2}$ can be generated by 
repeated differentiation of this expression. The result is the infinite sum

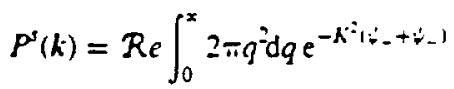

$$
\begin{aligned}
& \times \sum_{n=0}^{x} \frac{(-)^{n}(k q s / 2)^{2 n}}{n !^{2}}\left(\frac{\partial}{\partial B}\right)_{B=\mathcal{A}^{\prime} !}^{n} F(k q c . B),
\end{aligned}
$$

where $F$ is the function defined by equation (26), and $s$ and $c$ are given by equations (54) and (55). The sum in equation (A1) can be truncated to a few terms if kqs is small. For large $k$ (non-linear limit), this truncation effectively works for all $q$, since then the exponential factor $e^{-K^{\prime}\left(\psi_{-}+t_{-}\right)}$causes the integrand to become negligible when kgs is not small.

The expansion ( $A 1$ ) fails when kgs is large and at the same time $K^{2}\left(\psi_{+}+\psi_{-}\right)$is small. Here a series expansion in $K^{2} \psi_{-}$leads to the following infinite sum:

$$
\begin{aligned}
& P^{\prime}(k)=\operatorname{Re} \int_{0}^{x} 4 \pi q^{2} d q e^{-k^{2}+}
\end{aligned}
$$

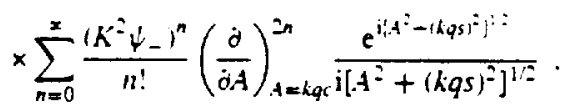

Expression (A?) works when $K^{2} \psi_{-}$is small and kqs is not small, which is complementary to the domain of validity of equation (A1). A program such as Matrematica helps considerably with the algebra involved in taking the repeated derivatives in both equations ( $A 1)$ and $\left(A_{2}\right)$.

The integration of equations ( $A 1)$ and (A2) over q must be done numerically. As in the unredshifted case, the oscillatory character of the integrand for large $k q$ can be converted into exponential decay by shifting the path of integration of $q$ into the upper complex plane. One should the careful here that, although the real parts of the integrands of equations (A1) and (A2) agree for real $q$, their imaginary parts differ, so that their analytic continuations in the complex $q$-plane diffet. The integrands could be made identical by adding their complex conjugate expressions, but this would introduce an undesirable exponentially increasing component in the upper complex plane. Therefore, if it is necessary to use both expressions ( $A 1$ ) and (A2) in integrating from $q=0$ to $x$, then the transition from one expression to the other along the path of integration must occur on the real line.

In the cases that we tried, an integration path similar to that described at the end of Section 2.2 proved satisfactory. Namely. integrate first some way along the real axis. then complete the integration along a straight line upwardly slanted in the complex plane. In the case $K^{2} \downarrow-(q=1 / k s) \geq 1$. equation (A1) works everywhere on the path of integration. In the case $K^{\prime} \psi_{-}(q=1 / k s)<1$, equation (A1) works for the first part of the integration. along the real line, but equation ( $A_{2}$ ) is required for the second part, a suitable path being vertically upuards in the complex plane.

Computing the quadrupole and monopole harmonics of the redshiff power spectrum requires a final numerical integration of $P^{\prime}(k)$ over $\mu_{k}$ with $P_{i}\left(\mu_{k}\right)$ for $\ell=0$ and 2 , equation (59). 